\title{
Poly(A) signal-dependent degradation of unprocessed nascent transcripts accompanies poly(A) signal-dependent transcriptional pausing in vitro
}

\author{
AMIR KAZEROUNINIA, ${ }^{1}$ BENSON NGO, ${ }^{1}$ and HAROLD G. MARTINSON ${ }^{1,2}$ \\ ${ }^{1}$ Department of Chemistry and Biochemistry, University of California at Los Angeles, Los Angeles, California 90095-1569, USA \\ ${ }^{2}$ Molecular Biology Institute, University of California at Los Angeles, Los Angeles, California 90095-1569, USA
}

\begin{abstract}
The poly(A) signal has long been known for its role in directing the cleavage and polyadenylation of eukaryotic mRNA. In recent years its additional coordinating role in multiple related aspects of gene expression has also become increasingly clear. Here we use HeLa nuclear extracts to study two of these activities, poly(A) signal-dependent transcriptional pausing, which was originally proposed as a surveillance checkpoint, and poly(A) signal-dependent degradation (PDD) of unprocessed transcripts from weak poly(A) signals. We confirm directly, by measuring the length of RNA within isolated transcription elongation complexes, that a newly transcribed poly(A) signal reduces the rate of elongation by RNA polymerase II and causes the accumulation of elongation complexes downstream from the poly(A) signal. We then show that if the RNA in these elongation complexes contains a functional but unprocessed poly(A) signal, degradation of the transcripts ensues. The degradation depends on the unprocessed poly(A) signal being functional, and does not occur if a mutant poly(A) signal is used. We suggest that during normal 3 '-end processing the uncleaved poly(A) signal continuously samples competing reaction pathways for processing and for degradation, and that in the case of weak poly(A) signals, where poly(A) site cleavage is slow, the default pathway to degradation predominates.
\end{abstract}

Keywords: poly(A) signal; transcriptional coupling; transcriptional pausing; cleavage and polyadenylation; poly(A) signaldependent RNA degradation; poly(A) signal-dependent surveillance

\section{INTRODUCTION}

The cleavage and polyadenylation reaction, which is responsible for defining the ends of most eukaryotic messenger RNAs, has emerged also as one of the central hubs for coordinating the transcription, splicing, release, transport, and surveillance of those same transcripts (Proudfoot et al. 2002; Nag et al. 2007; Perkins et al. 2008; Rigo and Martinson 2008; Rougemaille et al. 2008; Schmid and Jensen 2008). In keeping with this complexity of function, the apparatus designed to carry out cleavage and polyadenylation is also very complex-more than an order of magnitude larger and more complex, for example, than the capping apparatus (Pillutla et al. 1998; Shi et al. 2009), although cleavage/polyadenylation per se is chemically no more complicated than capping.

Reprint requests to: Harold G. Martinson, Department of Chemistry and Biochemistry, University of California at Los Angeles, Los Angeles, CA 90095-1569, USA; e-mail: hgm@chem.ucla.edu; fax: (310) 206-4038.

Article published online ahead of print. Article and publication date are at http://www.rnajournal.org/cgi/doi/10.1261/rna.1622010.
Assembly of the cleavage/polyadenylation apparatus appears to involve a delicately orchestrated sequence of events beginning, possibly, with the recruitment of some factors to the promoter (Dantonel et al. 1997; Calvo and Manley 2003; Glover-Cutter et al. 2008). The canonical mammalian poly(A) signal is characterized by two elements that flank the poly(A) cleavage site, the AAUAAA hexamer and a downstream G/U-rich region (Zhao et al. 1999). Almost immediately upon transcription of the poly(A) signal, the AAUAAA hexamer is bound by CPSF (Nag et al. 2007). Recruitment of CstF, which binds the G/U-rich region, appears to occur subsequently, and may be accompanied by substantial rearrangements among the components of the assembling apparatus (Nag et al. 2007). During this period, functional connections are established between the cleavage/polyadenylation apparatus and the upstream splicing apparatus (Rigo and Martinson 2008). Ultimately, the cleavage/polyadenylation apparatus becomes firmly attached to the polymerase as it commits to cleavage at the poly(A) site (Rigo et al. 2005; Rigo and Martinson 2008). It is possible that these culminating events coincide 
with the establishment of gene looping by the cleavage/ polyadenylation apparatus (Uhlmann et al. 2007; Perkins et al. 2008).

The first discernable consequence of transcription across the mammalian poly $(\mathrm{A})$ signal is a reduction in the rate of elongation by the polymerase due to the interaction of CPSF with the poly(A) signal hexamer (Orozco et al. 2002; Park et al. 2004; Nag et al. 2006, 2007). This poly(A) signaldependent pausing can be seen in the $3^{\prime}$ flanks of genes across the genome (Gromak et al. 2006; Boireau et al. 2007; Glover-Cutter et al. 2008; Lian et al. 2008) and has been proposed to coincide with checkpoint activity that leads either to cleavage at the poly(A) site or, alternatively, to continued transcription, or to degradation of the transcript (Orozco et al. 2002; Rigo et al. 2005; Nag et al. 2007). In yeast, surveillance during the polyadenylation phase of $3^{\prime}$-end processing is well documented for situations in which the polyadenylation has been compromised by defects in mRNP maturation factors or by the action of special regulatory elements (Schmid and Jensen 2008; Roth et al. 2009).

The efficiency of cleavage and polyadenylation is an important determinant of the overall level of gene expression in mammals, as in other eukaryotes. Thus, for similar rates of transcription, decreasing efficiencies of cleavage and polyadenylation give rise to decreasing final levels of gene expression (Edwalds-Gilbert et al. 1993; Chao et al. 1999; Wu and Alwine 2004). An unresolved question concerns the mechanism by which transcripts with weak poly(A) signals are discarded when they fail to get cleaved and polyadenylated. For example, the relatively weak SV40 early poly(A) signal supports mRNA production less than one-fifth as efficiently in mammalian cells as does the stronger SV40 late poly(A) signal, even when both are transcribed at the same rate (Carswell and Alwine 1989). Yet, although most transcripts carrying SV40 early poly(A) sites do not get processed at those sites, little or no RNA carrying uncleaved poly(A) sites can be found in the steadystate nuclear RNA from such cells (Connelly and Manley 1988; Chao et al. 1999; Park et al. 2004; Nag et al. 2006). Therefore, this unprocessed RNA is exceedingly unstable. Nevertheless, it is not merely the lack of processing at the poly(A) site that renders uncleaved RNA unstable, because full inactivation of the weak poly(A) signal, either mutationally or by using antisense, does lead to accumulation of uncleaved RNA in the nuclear compartment (Connelly and Manley 1988; Chao et al. 1999; Nag et al. 2006). Thus, paradoxically, the ability to discard unprocessed RNA appears to require the same weak poly(A) signal whose inefficient cleavage and polyadenylation was responsible for the lack of processing in the first place. Since weak poly(A) signals process more slowly than strong ones (Chao et al. 1999), this situation is suggestive of a surveillance function in which transcripts that are not processed quickly enter a poly(A) signal-dependent discard (PDD) pathway.
Here, we have sought to reproduce in vitro the poly(A) signal-dependent pause and the accompanying surveillance that may be responsible for the PDD of unprocessed transcripts bearing weak poly(A) signals. Although PDD is not evident in previous experiments involving weak poly(A) signals in vitro (Ryner et al. 1989; Prescott and Falck-Pedersen 1992), the processing in those experiments was not coupled to transcription. Therefore, since mRNA surveillance is thought to begin cotranscriptionally (Andrulis et al. 2002; Hieronymus et al. 2004), we decided to search for evidence of poly(A) signal-dependent transcript degradation using a transcription system that is capable of responding to the poly(A) signal in vitro (Tran et al. 2001). In this report we show that several aspects of the poly(A) signal-dependent pause are reproduced in vitro, and that coincident with or immediately following this transcriptional slowdown, PDD of nascent transcripts bearing uncleaved poly(A) signals becomes evident.

\section{RESULTS}

\section{The poly(A) signal directs the polymerase to pause in vitro}

To study poly(A) signal-dependent pausing in vitro (Fig. 1) we compared transcripts produced by polymerases that have crossed a functional poly(A) signal with transcripts produced by polymerases that have crossed an inactive, mutated poly(A) signal (Tran et al. 2001; Nag et al. 2006). Polymerases that have been slowed down by pausing will not transcribe as far during the time of the experiment, and can be identified by the shorter transcripts they carry. To ensure that any short transcripts detected in the analysis reflect slow polymerases, rather than terminated polymerases or poly(A) site-cleaved RNA, we analyzed only transcripts that were isolated from transcription elongation complexes (TECs) that had been separated from free RNA by size exclusion chromatography under strongly dissociating conditions $(1 \mathrm{M} \mathrm{NaCl}, 1 \%$ Sarkosyl). In addition, to be sure that any short transcripts detected in the analysis reflect slow polymerases rather than partial degradation of the RNA, we used a pulse-chase format (Fig. 1A). This made sure that any shortening of the TEC RNA due to degradation would simultaneously remove $\left[{ }^{32} \mathrm{P}\right]$ label, thereby reducing or eliminating the ability of the $5^{\prime}$ truncated RNA to contribute $\left[{ }^{32} \mathrm{P}\right]$ signal to the analysis. Finally, to focus on the transcriptional effects of the intact unprocessed poly(A) signal, we used the SV40 early poly(A) signal core, which is so weak in a coupled transcription/ processing system that hardly any processing occurs, though it nevertheless communicates well with the polymerase (Tran et al. 2001).

Figure 1B displays the results from such an experiment. In Figure $1 \mathrm{~B}$, lane 1, a region of intensity slightly above the position of the poly(A) site reveals an accumulation of 
A

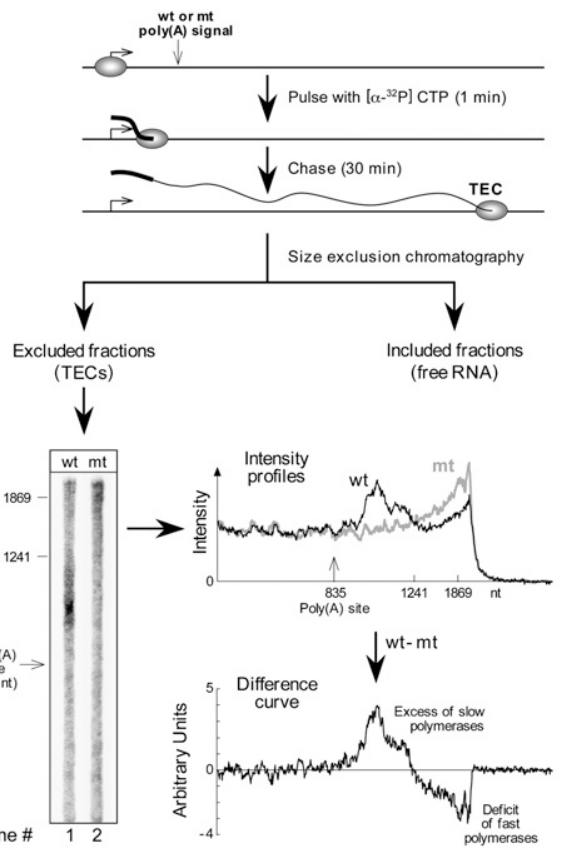

C
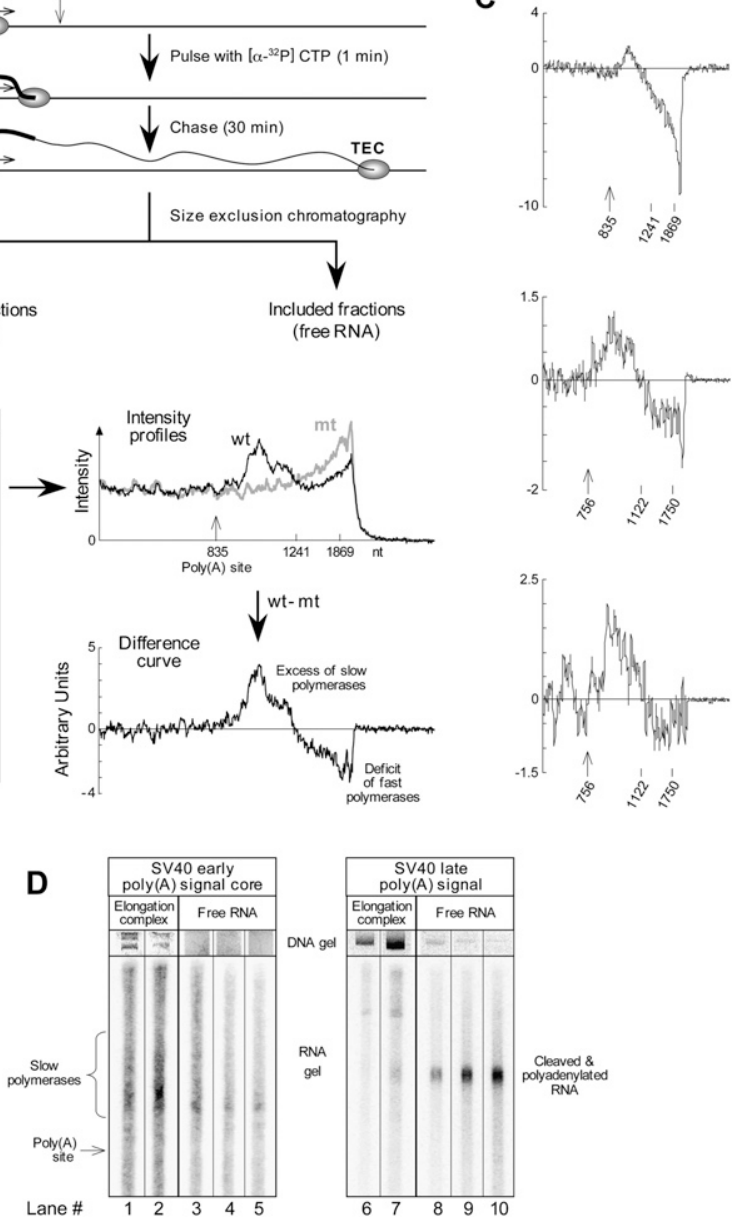

FIGURE 1. Survey of paused polymerases by transcript analysis. (A) Experimental protocol. DNA from Reporter 3 was transcribed in vitro using a pulse-chase format very similar to that of Rigo et al. (2005), and then TECs were isolated by size fractionation in the presence of $1 \mathrm{M}$ $\mathrm{NaCl}$ and $1 \%$ Sarkosyl. A fourfold increase over the typical reaction size was used. The length of the pulse was chosen to be short enough to preclude any polymerases from reaching the poly(A) site before the start of the chase. $(B)$ Comparison of TEC fractions for wild-type and mutant versions of the reporter. Size calibrations are based on RNA transcribed from the same or similar template in the presence of complementary DNA oligonucleotides that direct RNase $\mathrm{H}$ to cut the RNA at specified locations (Rigo et al. 2005). TECs (identified by the presence of plasmid DNA) eluted from the column in fractions 8 and 9 for the wild type and in fractions 7 and 8 for the mutant. Gel lanes 1 and 2 show column fractions 9 and 8 , respectively. However, the intensity profiles to the right of the gel show the summed intensities for both TEC fractions eluting from the column. In preparing these profiles, the data were background corrected and normalized, as described below, to control for variations in the amount of template used in the different transcription reactions and for variations in sample recovery. First, the background in the gel above the region containing RNA was subtracted from the signal in all lanes, bringing the far right of the line graphs to zero. Then, the remaining signal in each lane was normalized at each position to the sum of the signal for TECs that had not reached the poly(A) signal. Thus, the average signal to the left of the poly(A) site for each graph is identical, by definition - as it should be, since the wild-type and mutant templates are identical upstream of the poly(A) signal. The difference curve was calculated by subtracting the mutant intensity profile from the wild-type one and expressing the result in arbitrary units related to the fraction of all RNA produced in the experiment. $(C)$ Difference curves from three additional experiments. Reporters 3 and 2a were used for the top panel and for the bottom two panels, respectively. (D) Gel-electrophoretic analysis of RNA and DNA from size fractionated transcription reactions for poly(A) signals that either do not (lanes 1-5) or do (lanes 6-10) process efficiently in vitro. Fractions 8, 9, 11, 12, and 13 are shown for the SV40 early column, and fractions $7,8,10,11$, and 12 are shown for the SV40 late column. Transcription and processing of the SV40 late RNA was exactly as described in Rigo et al. (2005) with a 2-min pulse and a 13 -min chase. Lane 2 in $D$ is the same as lane 1 in $B$. polymerases a short distance downstream from the wild-type poly(A) signal. In contrast, Figure $1 \mathrm{~B}$, lane 2, shows no such accumulation downstream from a mutant poly(A) signal. Instead, many polymerases on this template have proceeded much farther downstream, producing RNAs that migrate in the poorly resolved upper region of the gel (Fig. 1B, lane 2). This provides the first direct evidence for substantial slowing by polymerases once they cross a poly(A) signal. Although we have not explicitly ruled out the formal possibility that the poly $(\mathrm{A})$ signal causes polymerases to arrest on the template, we will assume that this poly(A) signaldependent pausing is the same poly $(\mathrm{A})$ signal-dependent pausing that has been described in vivo. Note that the pulsechase format of the experiment shows that this effect on transcription is imposed by the poly(A) signal on the polymerase in the absence of cleavage at the $\operatorname{poly}(\mathrm{A})$ site (which would remove all label from the elongation complexes) (see Fig. 1A).

In the upper graph to the right of the gel in Figure 1B the intensities for both lanes on the gel are plotted as a function of distance and superimposed on each other after background subtraction and normalization, as described in the figure legend. The result shows clearly that, after crossing a poly(A) signal, many polymerases linger behind that would otherwise proceed rapidly down the template. The graph at the bottom of Figure 1B summarizes both the wildtype and the mutant results in a single difference curve, obtained by subtracting the mutant from the wild-type curve in the upper graph. This difference curve illustrates clearly the generation of slow polymerases at the expense of rapid ones downstream from a functional poly(A) signal.

The results of Figure $1 \mathrm{~B}$ are confirmed by difference curves from three additional independent experiments, as summarized in Figure 1C. Given the complexity of these experiments, it is not surprising that there is significant scatter in the relative sizes of the peaks and valleys of these curves. Nevertheless, 
in all cases there is a poly(A) signal-dependent increase in the number of slow polymerases downstream from the poly(A) signal, followed by a poly(A) signal-dependent deficit in the number of faster polymerases farther downstream.

To confirm that the material identified as paused RNA in Figure $1 \mathrm{~B}$ originated from elongation complexes and not from processed or partially processed RNA, we carried out an additional control. In Figure 1D the size exclusion data from the experiment that produced the SV40 early RNA of Figure 1B (lane 1) are compared directly with comparable data for RNA containing the strong SV40 late poly(A) signal, which processes efficiently in vitro (Rigo et al. 2005). The first two lanes of each panel in Figure 1D show the leading edge of the elongation complex fractions from the exclusion column (identified by the presence of plasmid DNA), and the remaining three lanes in each panel show the first few free RNA-containing fractions (see Nag et al. 2006 for further characterization of this column system). It can be seen that the SV40 early RNA (lanes 1-5) emerges predominantly in the elongation complex fractions with some trailing into the free RNA, while the processed SV40 late RNA (lanes 6-10) clearly fractionates like free RNA, with a little spreading into the proximal elongation complex fraction. Thus, the prominent region of RNA intensity centered at about $1 \mathrm{~kb}$ in the wild-type lane of Figure 1B does indeed consist predominantly of RNA from paused elongation complexes, and is not simply processed or partially processed RNA.

\section{Further analysis of poly(A) signal-dependent pausing}

The difference curves in Figure 1, B and C, are all in qualitative agreement that polymerases slow down after crossing a wild-type, but not a mutant poly(A) signal. However, the curves vary substantially in shape. We believe this most likely reflects sample-to-sample variations that are magnified when the intensities along one lane of a gel are subtracted from the intensities of another. We therefore decided to characterize poly(A) signal-dependent pausing also by a more quantitative approach that allowed the use of internal standards in all of the samples.

Figure $2 \mathrm{~A}$ outlines such an experiment. Templates bearing wild-type or mutant poly(A) signals were transcribed in separate reactions (together with a reference plasmid), and TECs were separated from free RNA by size exclusion chromatography. G-less cassettes in the template, positioned both next to the poly(A) signal (pre-cassette) as well as 1-kb farther downstream (post-cassette), permitted polymerase progress to be monitored quantitatively. A reduction in transcription velocity results in less postcassette being transcribed relative to pre-cassette, because fewer of the slow polymerases reach the post-cassette during the time allotted for transcription (Tran et al. 2001). To quantitate cassette transcription, the transcripts were digested with RNase T1 (which is G-specific) and the surviving G-less cassettes were resolved on a polyacrylamide gel (Fig. 2B).

The gel in Figure 2B shows that, relative to the 120nucleotide (nt) pre-cassette, significantly less 261-nt postcassette was transcribed from the template bearing a wildtype poly(A) signal (Fig. 2B, lane 1) than from the template with a mutant poly(A) signal (Fig. 2B, lane 2). This confirms that the polymerases slow down upon encountering the wild-type poly(A) signal, and are less likely to

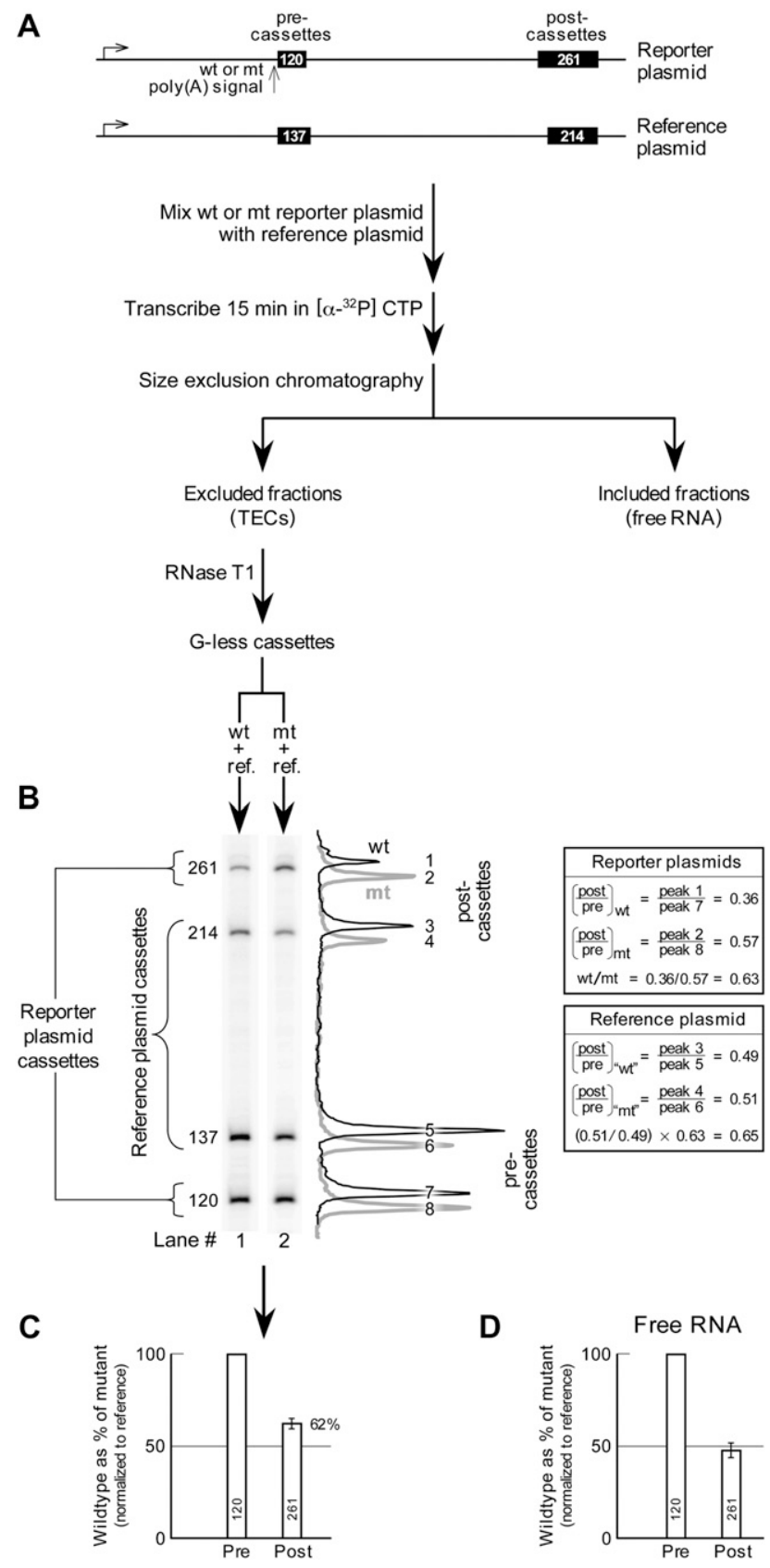

FIGURE 2. (Legend on next page) 
reach the post-cassette during the time of the experiment. The visual comparison in the gel of Figure $2 \mathrm{~B}$ was facilitated by adjusting the overall intensities in the two lanes to equalize the 120 -nt pre-cassette bands. This operation is formally equivalent to numerically normalizing the intensity of the post-cassette to that of the precassette in each lane prior to calculating the wild-type/ mutant ratio for the post-cassettes, as is done in the upper table to the right of the gel. As shown in this table, only $63 \%$ as many polymerases reached the post-cassette in this experiment after transcribing a wild-type as compared with a mutant $\operatorname{poly}(\mathrm{A})$ signal.

The gel lanes in Figure 2B also contain bands from the reference plasmid transcripts. Since the post/pre cassette ratios for transcripts from the reference plasmid should theoretically be identical in all reactions, these ratios can be used to correct the reporter plasmid numbers for sampleto-sample variation, as shown in the lower table to the right of the gel. The averaged results, which incorporate this reference correction, for two independent experiments are illustrated in Figure 2C. Here, we define the number of polymerases crossing the pre-cassette as $100 \%$ and then show that, on average, only $62 \%$ as many of these reach the postcassette when they carry a wild-type poly(A) signal ("wild-type" TECs) as when they carry a mutant poly(A) signal ("mutant" TECs).

It is formally possible that the deficit of wild-type TECs carrying post-cassettes results, not from pausing before they reach the post-cassette, but from release after they pass it. However, this would predict a corresponding increase in post-cassettes in the free wild-type RNA from the included fractions of the size exclusion chromatography, and Figure $2 \mathrm{D}$ shows that this is not the case. In fact, Figure $2 \mathrm{D}$ resembles Figure $2 \mathrm{C}$, suggesting that the free RNA may simply be a random sample of nascent cassette-containing RNA, which is released from the DNA by the poly(A)-

FIGURE 2. Poly(A) signal-dependent pausing in vitro on circular templates. $(A)$ Experimental protocol with plasmid templates drawn to scale. In vitro transcriptions for column separation were carried out as described previously (Nag et al. 2006) using Reporter 1. (B) Cassette analysis of the principal TEC column fraction (fraction 8) for a wild-type and a mutant transcription reaction. The signal intensities of the cassette bands in the wild-type and mutant gel lanes are presented as line graphs, which are offset slightly to enhance clarity. Some key ratios are given in the table. The "wt" and " $m$ " designations in the case of the reference plasmid cassette ratios refer to transcription mixtures where the reporter was either wild type or mutant (although the same reference plasmid was used for both). (C) Overall cassette analysis for the TEC fractions. Data were summed over the two TEC fractions 7 and 8 for the experiment in $B$, and the resulting reporter $\mathrm{wt} / \mathrm{mt}$ ratio was normalized to the reference $\mathrm{wt} / \mathrm{mt}$ ratio. The experiment was repeated, and the average and range of values obtained is shown. There is no error bar for the 120-nt cassette because normalization was to this cassette so that this value, by definition, is $100 \%$. $(D)$ Cassette analysis of a representative free RNA fraction 11 for the experiment in $B$, normalized to the reference and then averaged across two experiments as in $C$. independent transcript release factor TTF2 present in the extract (Liu et al. 1998; Hara et al. 1999).

We also examined poly(A) signal-dependent pausing by analysis of heparin and Sarkosyl-washed TECs isolated using immobilized templates rather than size exclusion chromatography (Fig. 3). Although the magnitude of the poly(A) signal-dependent pausing response, like cleavage and polyadenylation itself (Yonaha and Proudfoot 2000; Rigo et al. 2005), is reduced on immobilized templates, these experiments confirm that TECs proceed more slowly when they carry a wild-type $\operatorname{poly}(\mathrm{A})$ signal than when they carry a mutant poly(A) signal (Fig. 3B,C).

\section{Pausing in vitro occurs independently of DNA sequence downstream from the poly(A) signal}

Poly(A) signal-dependent termination can occur independently of sequence downstream from the poly(A) signal (Yeung et al. 1998). To establish whether poly(A) signaldependent pausing also is independent of downstream sequence, the various pausing experiments in Figures 1 and 3 were carried out using two different reporters having completely unrelated sequences between their cassettes. Thus, in the case of the reporter used for Figure $1 \mathrm{~B}$ and the top frame of Figure 1C, the slow TECs accumulate over an arbitrarily chosen eukaryotic sequence, whereas for the reporter used for the bottom two frames of Figure $1 \mathrm{C}$ they accumulate over prokaryotic DNA. Similarly, in the cassette analyses of Figure 3, wild-type TECs exhibit a clear delay in reaching the second cassette, regardless of whether the intercassette region consists of eukaryotic (Fig. 3B) or prokaryotic (Fig. 3C) DNA. The slightly reduced effect seen in Figure 3C presumably reflects the somewhat shorter distance separating the cassettes in the reporter used for those experiments (see Fig. 3A; Tran et al. 2001).

\section{Involvement of the SV40 early poly(A) signal in a nascent transcript discard pathway}

As noted in the Introduction, weak poly(A) signals, whose processing is slow, direct not only processing (at a low level), but also the degradation of those transcripts not destined to be processed. Since recapitulation of PDD by weak poly $(A)$ signals in vitro may require the use of a transcriptionally coupled system (see Introduction), we turned our attention to the possibility that poly $(\mathrm{A})$ signalcoupled surveillance may accompany the poly(A) signaldependent transcriptional pause described here.

Studying specific degradation events in a transcriptionally coupled system requires the ability to detect and quantitate transcript loss from a highly heterogeneous population and to mitigate or control for changes in the transcript population that arise from new transcription, pausing, or processing itself. The approach described in Figures 2 and 3 , in which the SV40 early poly(A) signal was used in 
A

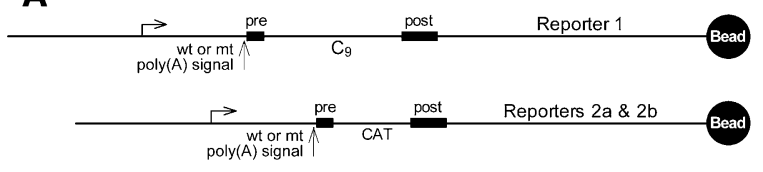

B
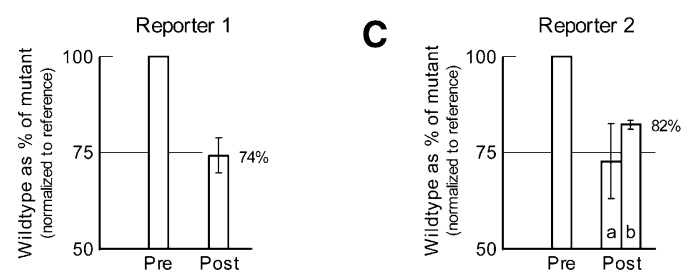

FIGURE 3. Poly(A) signal-dependent pausing in vitro on immobilized templates. (A) The immobilized reporter DNAs drawn to scale. Transcription was carried out as for Figure 2. (B) Cassette analysis for washed TECs isolated using Reporter 1 . The average and standard deviation for three independent experiments is shown. (C) Cassette analysis for washed TECs isolated using Reporters 2a and 2b (independently cloned, presumably identical plasmids; see Materials and Methods). The average and range for two independent experiments is shown for reporter $2 \mathrm{a}$, and the average and standard deviation for three independent experiments is shown for reporter $2 \mathrm{~b}$. Note that four out of the five results obtained clustered around the value of $82 \%$. We consider the one low value (the $63 \%$ for reporter $2 a$ ) to be an outlier.

conjunction with G-less cassettes, appeared to be an appropriate point of departure for several reasons. First, the SV40 early poly(A) signal is weak in vivo (Carswell and Alwine 1989; Chao et al. 1999) and in vitro (Rigo et al. 2005). Second, the almost complete absence of processing under the conditions used here minimizes cleavage and polyadenylation as a competing process to be quantitated during the analysis. Third, the use of G-less cassettes allows the quantitation of discrete bands on a gel despite the heterogeneity of the transcript population. Finally, transcription and pausing are easily eliminated as variables by stopping transcription with $\alpha$-amanitin and then measuring the amount of transcript remaining as a function of time.

To quantitate the amount of transcript present, we used the G-less cassettes carried by the reporter plasmid shown in Figure 4A. All transcripts, both short and long, contained a 76-nt cassette near the $5^{\prime}$-end. In addition, 120-nt and 261-nt cassettes appeared only in transcripts long enough to contain a poly(A) signal. However, transcription time was minimized so that most transcripts (about two-thirds) remained too short to contain a poly(A) signal. This allowed us to express the amount of poly(A) signal-containing transcripts (containing the 120-nt and the 261-nt cassettes) as a fraction of the total population of transcripts (the 76-nt cassettes), most of which lacked a poly(A) signal. Expressing the amount of poly(A) signal-containing transcripts as a ratio in this way eliminated various uncertainties in the data, such as the efficiency of sample recovery. To extract poly(A)-specific information we further normalized all data for the wild-type poly(A) signal to data obtained in parallel using a mutant poly(A) signal. PDD is indicated by a decrease in this ratio as a function of time.

The gel in Figure 4B shows the cassette bands from an experiment designed to detect PDD. Since the short transcription time allowed only $5 \%$ of the polymerases to reach the 261-nt cassette, its signal is comparatively weak, and an enhanced version of the 261-nt cassette panel is included in the figure. Note that while there is obvious poly(A) signal-dependent pausing (deficit of 261-nt cassettes in the wild-type lanes, compared with the mutant), this does not affect the analysis of PDD, because the pausing reflects events that occurred before transcription was stopped, prior to the zero-time reference point for PDD (see Fig. 4A). PDD is then revealed by the comparison between 0 and $19 \mathrm{~min}$. For example, the intensity of the 120-nt cassette band in Figure 4B decreases more for the wild type than for the mutant between 0 and $19 \mathrm{~min}$. Quantitative analysis (beneath the gel) shows that the wildtype/mutant ratios for both the 261-nt and the 120-nt cassettes decrease significantly in this time interval (from 0.40 to 0.29 and from 0.95 to 0.61 , respectively), indicating the preferential degradation of one-quarter to one-third of the transcripts bearing wild-type versus mutant poly(A) signals. Note that this degradation is in addition to the obvious poly(A)-independent degradation displayed by the 76-nt cassette (Fig. 4B, cf. lanes 1,2 and lanes 3,4), which has been normalized out in the analysis below the gel. The results from several such experiments are summarized in Figure 4C.

Because of the superficial similarities between the G-less cassette assays for poly(A) signal-dependent pausing (Figs. 2,3) and for PDD (Fig. 4), we want to emphasize the substantial differences between them. Thus, whereas the pausing assay measures primarily ongoing transcription, and reveals diminished transcription of the 261-nt cassette relative to the 120-nt cassette (Figs. 2, 3), the PDD assay measures only the degradation that occurs after transcription has been stopped and detects similar losses for both of the cassettes downstream from the poly(A) signal (Fig. 4C). The simultaneous loss of both of these cassettes from a population of unprocessed transcripts in a poly(A) signal-dependent fashion suggests the existence of a discard pathway in which the entire unprocessed transcript is eliminated.

\section{Pausing alone does not lead to discard}

We have previously shown that the poly(A) signal hexamer alone, like the SV40 early poly(A) signal (under the conditions used here), triggers pausing but cannot be processed (Nag et al. 2006). We wondered if it is simply the pausing, when not followed by processing, that triggers degradation of unprocessed transcripts. To answer this question we used a construct in which the SV40 early poly(A) signal had been replaced by a sequence containing 
A

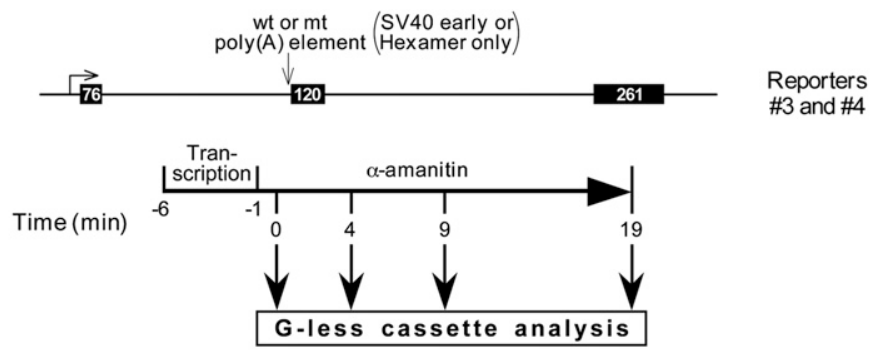

B

SV40 Early (Reporter \#3)

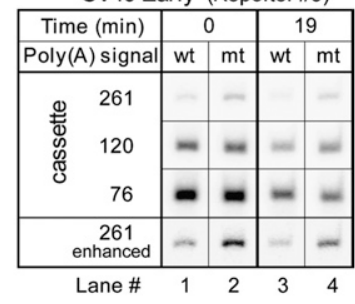

C

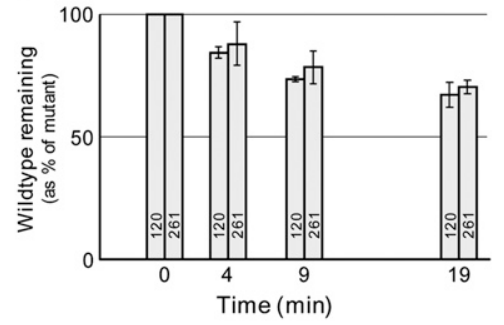

Normalization $\{261 / 76 \rightarrow 0.048 \quad 0.12 \quad 0.046 \quad 0.16$

to 76 cassette $\{120 / 76 \rightarrow \quad 0.45 \quad 0.48 \quad 0.39 \quad 0.64$

Wildtype/mutant $\left\{\begin{array}{lll}261 \mathrm{wt} / \mathrm{mt} & 0.40 & 0.29\end{array}\right.$

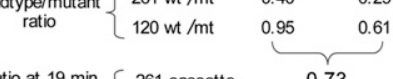

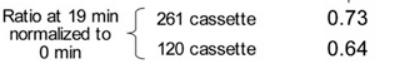

D

Hexamer only (Reporter \#4)

\begin{tabular}{|c|c|c|c|c|}
\hline \multirow{2}{*}{$\begin{array}{c}\text { Time }(\min ) \\
\text { Hexamer }\end{array}$} & \multicolumn{2}{|c|}{0} & \multicolumn{2}{|c|}{19} \\
\hline & wt & $\mathrm{mt}$ & wt & $\mathrm{mt}$ \\
\hline 261 & $=$ & - & & - \\
\hline $\begin{array}{ll}\Phi & 120 \\
\text { \& } & 120\end{array}$ & $=$ & $=$ & $=$ & $=$ \\
\hline 76 & $=$ & $=$ & $=$ & $=$ \\
\hline $\begin{array}{c}261 \\
\text { enhanced }\end{array}$ & $=$ & - & - & - \\
\hline Lane \# & 1 & 2 & 3 & 4 \\
\hline
\end{tabular}

E

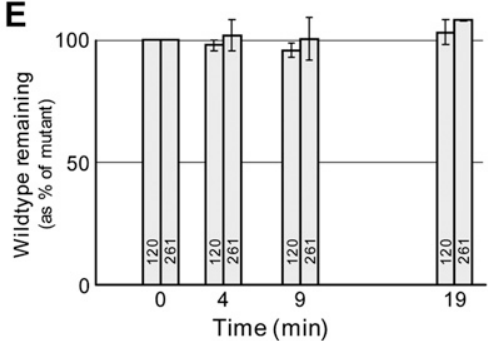

FIGURE 4. Poly(A) signal-dependent degradation in vitro. (A) Reporter construct drawn to scale, and the experimental protocol. Transcription was carried out according to Nag et al. (2006) with minor variations. The reaction was scaled up fivefold and transcription was stopped after $5 \mathrm{~min}$ by adding $5 \mu \mathrm{L}$ of $0.5 \mu \mathrm{g} / \mu \mathrm{L} \alpha$-Amanitin. At the time intervals indicated, $13.5 \mu \mathrm{L}$ aliquots were withdrawn and digested with RNase T1 in the presence of EDTA. $(B) \mathrm{A}$ gel showing the G-less cassette bands from the 0- and 19-min time points of an SV40 early PDD analysis. $(C)$ A quantitative summary of several SV40 early PDD analyses, calculated as shown beneath the gel of part $B$. The averages and standard deviations for the 19-min time point are from six independent experiments, and for the 5- and 9-min time points from four independent experiments. The averages include data from both Reporters $3 \mathrm{a}$ and $3 \mathrm{~b}$ (independently cloned, presumably identical plasmids; see Materials and Methods). (D) A gel showing the G-less cassette bands from the 0- and 19-min time points of a PDD analysis of Reporter 4, which contains two AATAAA hexamers in place of a poly(A) signal. (E) A quantitative summary showing the averages and ranges from two independent experiments. The range for the 261-nt cassette at 19 min was too small to be visible in the graph.

two AATAAA hexamers instead of a hexamer and a G/Trich region (Nag et al. 2006). Figure $4 \mathrm{D}$ shows the relevant bands from an experiment, like that of Figure 4B, designed to detect hexamer-dependent degradation using this hexamer-only construct. Although the gel confirms hexamer-dependent pausing (less 261-nt cassette in the wild-type lanes than in the mutant lanes), there is no evidence of any time-dependent loss of cassettes in a hexamer- dependent (i.e., wild-type versus mutant) fashion. The averaged results from this and one other experiment are summarized in Figure 4E. The contrast between the hexamer-only element (Fig. $4 \mathrm{D}, \mathrm{E})$ and the SV40 early poly (A) signal (Fig. 4B,C) is clear-while both cause pausing (albeit less for the hexameronly element in these experiments), only the poly(A) signal causes wildtype-specific degradation of transcripts. Therefore, pausing per se, not followed by processing, is insufficient to trigger the discard of unprocessed transcripts. Instead, it is the failure of some subsequent event in the processing pathway that leads to rejection of the transcript.

\section{Canonical poly(A) site cleavage does not precede discard}

There are at least three distinguishable events that follow pausing in the canonical 3 '-end processing pathway, whose failure could conceivably lead to degradation. These are cleavage at the poly(A) site, initiation of poly(A) tail synthesis, and the elongation phase of poly(A) tail growth (Wahle and Kühn 1997). In yeast, pre-mRNA is shunted to a degradation pathway in the presence of certain mRNP maturation factor mutations that affect the elongation phase of polyadenylation. These mutations apparently have little effect on cleavage at the poly(A) site (Saguez et al. 2008). We decided to test whether impaired poly(A) tail growth might similarly account for the tendency of pre-mRNA with weak poly(A) signals to be degraded rather than processed when processing is coupled to transcription.

If differences in the efficiency of poly(A) tail elongation, not differences in the efficiency of poly(A) site cleavage, are what account for the predominance of processing for strong, but the predominance of discard for weak poly(A) signals, then complete elimination of the elongation phase of poly(A) tail growth for both weak and strong poly(A) signals should eliminate the distinction between them. The ATP analog, $3^{\prime}$-dATP, can be used to prevent the addition of all but the first adenine of the poly(A) tail. If impaired poly(A) tail growth is what leads to degradation of RNA with weak poly(A) signals, then one might expect a strong poly(A) 
signal to similarly be degraded if poly(A) tail growth is blocked using $3^{\prime}$-dATP. In this case, both weak and strong poly(A) signals would produce low yields of cleaved RNA, bearing a single $3^{\prime}$-dATP at the $3^{\prime}$-end. Alternatively, if the appended $3^{\prime}$-dATP itself inhibits degradation in some way, perhaps because it prevents the residual synthesis of short oligo(A) tails that may be required for the degradation (Saguez et al. 2008), or by failing to support access to a $3^{\prime} \rightarrow 5^{\prime}$ exonuclease, then both weak and strong poly(A) signals should produce high yields of cleaved RNA. Note that by either scenario the yield of cleaved but not polyadenylated RNA is expected to be the same for both weak and strong poly(A) signals.

Figure 5A shows the procedure, and Figure 5B the results for such an assay. The incubation time in $\alpha$-amanitin (and $3^{\prime}$-dATP) was $50 \%$ longer than that used for Figure 4 to ensure that some processing would be detectable even for the weak SV40 early poly(A) signal. The transcript analysis in Figure $5 \mathrm{~B}$ shows that the difference in processing strength (eight- to ninefold) between the weak SV40 early and the strong SV40 late poly(A) signals is not abrogated by the presence of 3'-dATP; cleavage at the SV40 early poly(A) site is barely detectable (Fig. 5B, lane 2), whereas cleavage at the SV40 late site is robust (Fig. 5B, lane 5). Therefore, lack of poly(A) tail growth does not shunt the SV40 late RNA into the discard pathway, nor does the lack of residual oligo(A) tail synthesis allow the SV40 early poly(A) signal to produce large amounts of RNA. This is consistent with our previous finding that the use of $3^{\prime}$-dATP does not interfere with coupled processing (Rigo et al. 2005; Rigo and Martinson 2008, 2009).

The results of Figure 5B show that the comparative weakness of the SV40 early poly(A) signal is not attributable to a deficiency in the elongation phase of poly(A) tail growth. Therefore, the deficiency that triggers degradation is either in the poly(A) site cleavage step or in the ability to add the first adenine to the new $3^{\prime}$-end generated by the cleavage. To distinguish between these possibilities we compared the degradation of downstream RNA using aliquots of the same samples used for Figure 5B. Canonical poly(A) site cleavage is followed by $5^{\prime} \rightarrow 3^{\prime}$ exonucleolytic degradation of the downstream RNA by Xrn2 (Kaneko et al. 2007). If the reason for the weakness of the SV40 early poly(A) signal is a deficiency in the ability to add the first adenine to the upstream RNA following cleavage (rather than a deficiency in cleavage itself), then the poly(A) signaldependent degradation of downstream RNA observed in Figure $4 \mathrm{C}$ would be attributable to the Xrn2 exonucleolysis that follows cleavage at the poly(A) site, just as for a strong poly(A) signal. According to this scenario, degradation of downstream RNA for a weak poly(A) signal would differ little from that for a strong poly(A) signal.

In contrast, as judged by recovery of the 120-nt cassette, the results shown in Figure 5C indicate that the RNA immediately downstream from the strong SV40 late poly(A)
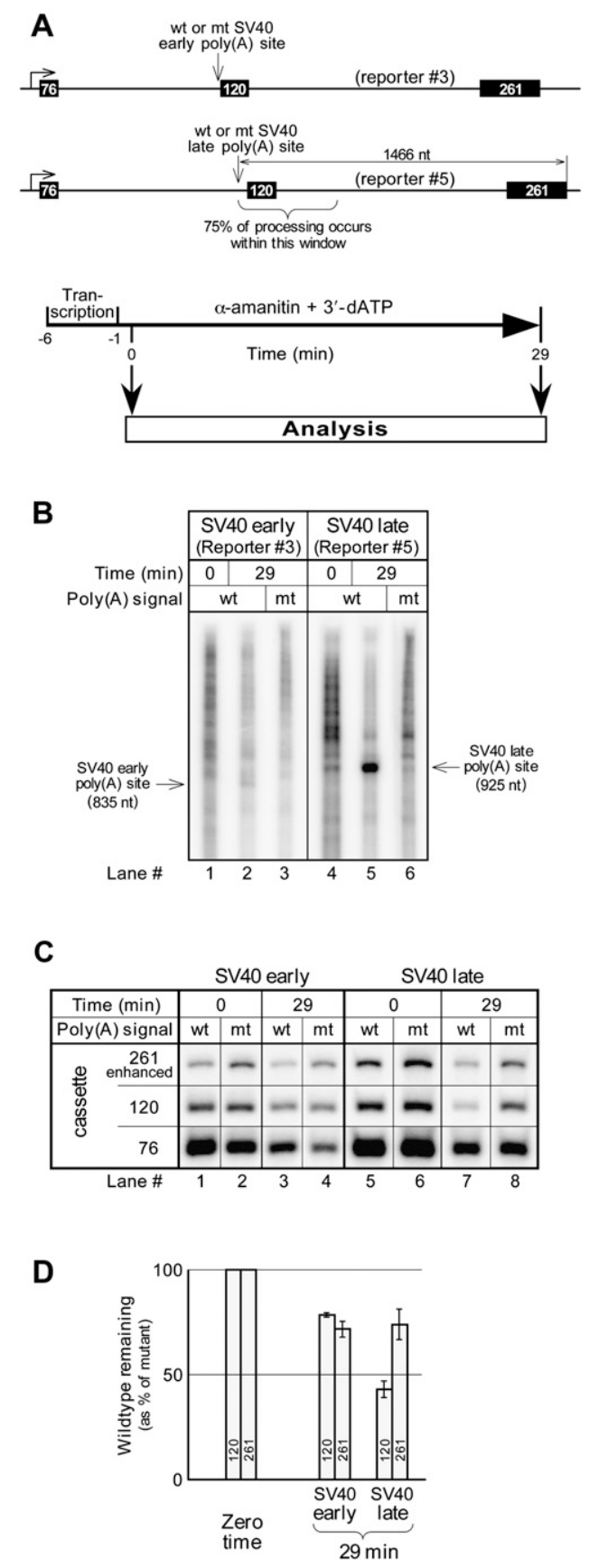

FIGURE 5. Poly(A) signal-dependent degradation in the presence and absence of processing. (A) Reporter constructs drawn to scale, and the experimental protocol. (B) Direct analysis of transcripts on a gel. (C) G-less cassettes from the transcripts. (D) A quantitative summary, calculated as for Figure $4 \mathrm{C}$, showing the averages and ranges from two independent experiments. For purposes of normalization to the 76-nt cassette, we assume the stability of cleaved, but nonpolyadenylated RNA is similar to that of uncleaved RNA in our system.

signal (Fig. 5C, lane 7) is degraded to a significantly greater extent than the RNA immediately downstream from the weak SV40 early poly(A) signal (Fig. 5C, lane 3). The averaged results from Figure $5 \mathrm{C}$ and a second independent 
experiment are summarized in Figure 5D, which was calculated as shown in Figure 4B. The quantitation confirms that substantially more RNA degradation occurs immediately downstream from the SV40 late poly(A) signal (as indicated by the 120-nt cassette) than occurs at any distance downstream from the SV40 early poly(A) signal. This more efficient degradation is correlated with more efficient cleavage at the SV40 late poly(A) site, and can therefore be attributed to Xrn2. Conversely, the much lessefficient degradation downstream from the SV40 early poly (A) site suggests strongly that cleavage is inefficient for this $\operatorname{poly}(\mathrm{A})$ signal, and that it is inefficient cleavage, rather than efficient $3^{\prime} \rightarrow 5^{\prime}$ degradation of efficiently cleaved RNA, which accounts for the low recovery of cleaved RNA in Figure 5B, lane 2.

We hypothesize that inefficient cleavage accounts for the inefficient processing at the SV40 early poly(A) site and that uncleaved RNA, waiting to be processed, is at risk of entering a discard pathway that does not depend on cleavage at the $\operatorname{poly}(\mathrm{A})$ site. Initial support for this idea comes, as discussed above, from a comparison of 120-nt cassette degradation rates downstream from the SV40 early and the SV40 late poly(A) sites. However, the idea is strengthened and extended by a consideration of the SV40 late poly(A) signal alone, and the differing fates of the 120-nt and 261-nt G-less cassettes in that construct (Fig. 5D). We have shown previously, in experiments like those of Figure 5B, that the probability of $3^{\prime}$-end processing progressively decreases for transcription complexes halted by $\alpha$-amanitin at increasing distances beyond the poly(A) signal (Rigo et al. 2005). Nearly 75\% of the transcription complexes that are destined to cleave their RNAs at the poly(A) site are found within the first 400 base pairs downstream (Rigo et al. 2005). Extrapolation of the data indicates that few if any of the transcription complexes that reach the end of the 261-nt G-less cassette before the $\alpha$-amanitin is added engage in processing. Therefore, SV40 late transcripts containing the 261-nt G-less cassette, like all SV40 early transcripts, have little chance of entering the processing pathway and are expected to be subject to PDD. Accordingly, degradation of the long SV40 late transcripts (containing the 261-nt cassette) is the same as for all SV40 early transcripts (Fig. 5D), suggesting that all poly(A) signals are endowed with the same discard capability, but differ in their ability to process.

\section{The discard pathway operates at the level of the transcription elongation complex}

The assays of Figures 4 and 5 were conducted on unfractionated transcription reaction mixtures. If PDD is coupled to transcription and is directed at uncleaved RNA waiting to be processed, it should be detectable by analysis of purified TECs. We therefore repeated the transcript degradation analysis of Figure $4, \mathrm{~B}$ and $\mathrm{C}$ using size exclusion chromatography (see Fig. 6A). We also analyzed the degradation results using two completely independent methods of normalization (Fig. 6D). This more rigorous

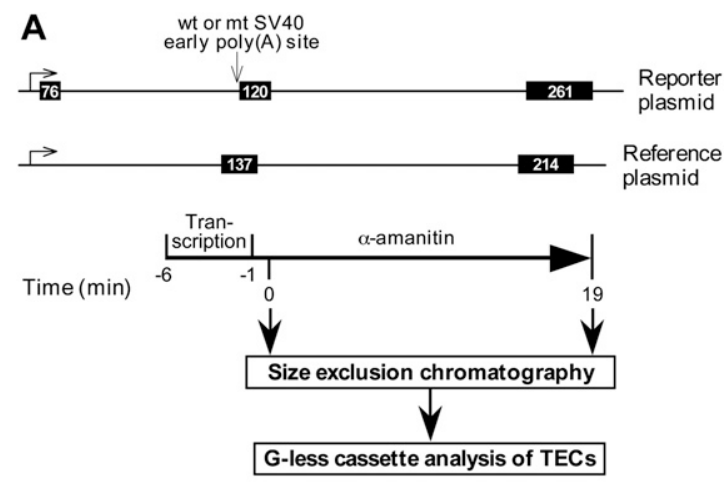

B

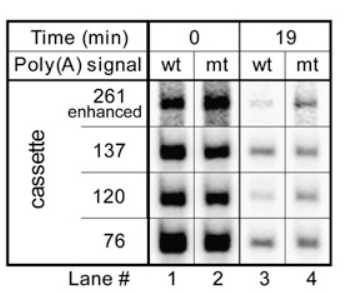

C Pausing $\left(\begin{array}{c}\text { TECs at } \\ \text { zero time }\end{array}\right)$
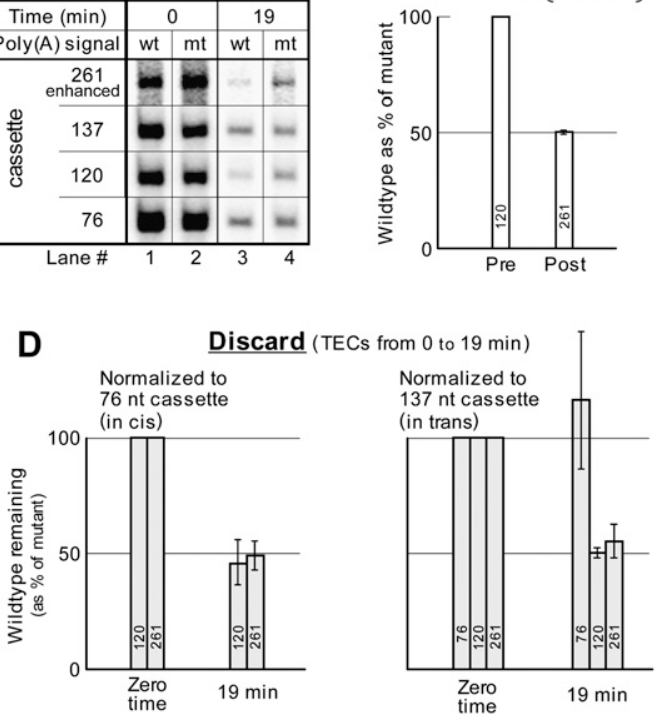

FIGURE 6. Poly(A) signal-dependent degradation is coupled to transcription. $(A)$ Reporter 3 and the reference plasmid drawn to scale, together with the experimental protocol. (B) G-less cassettes from the transcripts in the peak fraction of the column excluded volume. $(C)$ Pausing analysis at the zero time point of the TECs in the principal two fractions of the column excluded volume, showing the average and range from two independent experiments. Cassette analysis was as for Figure $2 \mathrm{C}$ but without normalization to the transcripts from the reference plasmid, because the 214-nt cassette band had insufficient counts for quantitation in one of the experiments. (D) PDD analysis of TECs at the 19-min time point. Two types of normalization are shown. Either the two downstream cassettes from the reporter transcripts were normalized to the 76-nt cassette as in Figure 4, or all three reporter cassettes were normalized to the 137nt reference cassette. The average and range for two independent experiments is shown. Note that whereas the wild-type/mutant ratio for the 261-nt cassette is less than that for the 120-nt cassette in $C$, the two cassettes are shown as being the same at zero time (both 100\%) in $D$. This is simply a consequence of our standard transcript degradation analysis, for which the 120-nt and the 261-nt cassette levels at all time points are normalized to the respective levels for these cassettes found at the 0 time point (see Fig. 4B). Thus, the equal zero time values in $D$ simply reflect self-normalization at this time point. 
elongation complex analysis confirmed the results of Figure 4B. First, there was strong poly(A) signal-dependent pausing at the zero time point. This is apparent from lanes 1 and 2 of Figure 6B, where the intensity of the 261-nt band, as compared with the 120-nt band, is less for the wild type than for the mutant (see quantitation in Fig. 6C). Second, between the zero time point and $19 \mathrm{~min}$, there is substantial PDD. This can be seen in Figure 6B, where the intensities of the 120-nt and the 261-nt cassette bands decrease much more for the wild type than for the mutant (see quantitation in Fig. 6D). These results confirm that the PDD pathway operates on TECs, presumably through surveillance of the nascent transcripts. Significantly, appreciably more PDD is observed in assays on purified TECs (Fig. 6) than in assays of the total transcription reaction mixture (Figs. 4, 5). This may reflect the presence in the total reaction mixture of TTF2-released RNA (see above), which, being uncoupled from transcription, is no longer subject to the PDD pathway.

Two different methods of standardizing the data have been used in this study. For Figures 2 and 3 the standard was provided in trans, and was a reference plasmid included in the reaction mixture. For Figures 4 and 5 the standard existed in cis, being a 76-nt G-less cassette adjacent to the promoter of the reporter plasmid itself. Although the cis location of the 76-nt cassette was considered desirable, the proximity to the promoter led to abortive elongation (Marshall and Price 1992; Orozco et al. 2002; Park et al. 2004). This is reflected in the 261/76 and the 120/76 ratios for the mutant templates in Figure 4B, which both increased significantly between 0 and $19 \mathrm{~min}$ (importantly, these ratios did not increase for the wild-type template, on account of PDD of the 261-nt and 120-nt cassettes). Although the poly(A)-independent degradation of the 76-nt cassette is theoretically normalized out by the taking of wild-type/mutant ratios, we nevertheless sought to increase our confidence in the final results by designing the experiment of Figure 6 to allow the use of both normalization approaches in parallel. Figure 6D shows that the extent of PDD is statistically identical whether normalization is to the 76-nt cassette in cis or to the 137-nt cassette in trans. This not only strengthens our confidence in the PDD results overall, but also provides strong experimental validation of both normalization procedures. Moreover, normalization of the 76-nt cassette itself to the 137-nt reference cassette in Figure 6D, right, confirms that the 76-nt cassette undergoes little or no PDD—as expected for TECs that have mostly not reached the poly(A) signal.

\section{Technical considerations}

We emphasized earlier that transcriptional pausing cannot contribute in any way to the results of a poly(A) signaldependent transcript degradation experiment, because the latter experiments were initiated only after blocking transcription with $\alpha$-amanitin. However, we now evaluate the converse possibility that PDD may contribute to the results of a G-less cassette pausing experiment. Although, in the absence of transcription, PDD affects both the 120-nt and the 261-nt cassettes similarly, it is conceivable that during active transcription PDD might affect the 261-nt cassette preferentially, and thereby give results similar to poly(A) signal-dependent pausing. This possibility exists because, when transcription is ongoing, the poly(A) signal in transcripts long enough to carry a 261-nt cassette has spent more time at risk for degradation than a poly(A) signal in shorter transcripts carrying only the 120 -nt cassette. The following analysis suggests that this possibility contributes, at most, minimally to our cassette-based measurements of pausing.

Figure 6D reveals about 50\% PDD after $19 \mathrm{~min}$ of incubation. Yet, Figure 6C shows pausing of the same magnitude, resulting from only $5 \mathrm{~min}$ of transcription-which includes the time taken for the polymerases to reach the poly(A) signal in the first place (about $2 \mathrm{~min}$ ) (Tran et al. 2001; Rigo et al. 2005). Thus, there is insufficient time for differential PDD to make more than a minor contribution to the 261-nt cassette deficit that we attribute to pausing. Moreover, if there is a lag before the start of PDD, as there is before cleavage and polyadenylation (Rigo et al. 2005), then the putative degradation may not contribute at all to pausing measurements after times of transcription as short as those in Figures 4-6. Finally, note that this analysis applies only to the quantitative pausing measurements carried out using G-less cassettes. The accumulation of slow polymerases seen by direct observation of intact transcripts (Fig. 1B) cannot be accounted for, or contributed to, in any way by PDD.

\section{DISCUSSION}

Almost immediately upon emerging from the mammalian RNA polymerase II in vivo, the poly(A) signal directs the polymerase to pause (Orozco et al. 2002; Nag et al. 2007). This pausing appears to be initiated well before the recruitment of the full cleavage and polyadenylation apparatus (Nag et al. 2007), probably during the lag that precedes 3 '-end processing (Rigo et al. 2005). We have suggested that this pause serves as an important node for surveillance activity, determining the fate of the transcript (Orozco et al. 2002; Nag et al. 2007). One manifestation of surveillance appears to be the transcript degradation that accounts for the reduced mRNA output from pre-mRNAs bearing weak poly(A) signals. This degradation depends on the poly(A) signal itself (see Introduction) and is most easily imagined as being in competition with authentic cleavage and polyadenylation. Thus, strong poly(A) signals process quickly (Chao et al. 1999) and produce much mRNA, whereas weaker poly(A) signals process more slowly, 
allowing many transcripts to be diverted to the discard pathway.

Here, we have studied pausing and discard using an in vitro system in which both of these activities are coupled to transcription. The studies were facilitated by use of a relatively weak poly(A) signal, which displays only moderate processing activity in vivo, and which is hardly processed at all under the in vitro conditions used here. This allowed us to visualize pausing and discard without significant interference by simultaneous processing. Figure 1B demonstrates directly that TECs pause after crossing a poly(A) signal, and Figure 6D shows that the transcripts associated with such complexes are then degraded in a poly(A) signaldependent manner with a half-life of $<20 \mathrm{~min}$.

\section{Poly(A) signal-dependent pausing of transcription}

The experiments of Figures 1-3, all based on isolated TECs, confirm known properties of poly(A) signal-dependent pausing determined in vivo and provide new insights. First, poly(A) signal-dependent pausing depends on an intact AAUAAA hexamer in the poly(A) signal (Figs. 1-3), consistent with the central role of CPSF in pausing (Nag et al. 2007). Second, the poly(A) signal does not act like a pause site, or blockade, to polymerase progress. That is, polymerases do not pause at the poly(A) signal itself but, consistent with a mechanism in which the elongation properties of the polymerase have been modified, the polymerases exhibit reduced elongation rates over the DNA downstream. Third, although poly(A) signal-dependent pausing occurs over downstream DNA, the pausing requires only the poly(A) signal and does not depend on any sequence elements in the downstream DNA (Figs. 1B,C, 3). Fourth, pulse-chase experiments show that the paused elongation complexes carry RNA that is not cleaved at the poly(A) site (Fig. 1). Therefore, pausing does not require processing, consistent with the appearance of pausing in the lag that precedes processing in coupled transcription-processing reactions using strong poly(A) signals in vitro (e.g., early time points of Fig. 1A in Rigo et al. 2005).

Finally, the modified properties of the TEC responsible for pausing appear to be transient. This is evident in the difference curves of Figure $1, \mathrm{~B}$ and $\mathrm{C}$, that reveal a concentration of slow polymerases immediately downstream from the poly(A) signal, which dissipates after several hundred base pairs under these in vitro conditions. The resumption of full-speed transcription may reflect the disruption of the CPSF-polymerase interaction (the initial cause of pausing) that has been proposed to occur during maturation of the cleavage and polyadenylation apparatus (Nag et al. 2007). The reduced number of paused elongation complexes at distal positions on the template in Figure 1 may also reflect the increasing effects of PDD on longer transcripts that have spent more time at risk. Interestingly,
poly(A) signal-dependent pausing can extend considerably beyond a few hundred base pairs in vivo (e.g., Gromak et al. 2006; Kaneko et al. 2007; Glover-Cutter et al. 2008), especially if either the CTD or the poly(A) signal has been compromised by mutation (Park et al. 2004; Nag et al. 2006, 2007). Since the polymerase and the poly(A) signal are in continuous communication as elongation proceeds (Kim and Martinson 2003), various elements downstream from the poly(A) signal would be expected to modulate poly(A) signal-dependent pausing through effects on the CTD and/or on the assembling cleavage and polyadenylation apparatus.

\section{Poly(A) signal-dependent degradation of nascent transcripts}

The experiments of Figures 4 and 5 reveal a new activity of the mammalian poly(A) signal-PDD of nascent transcripts. This activity was anticipated because inactivation of weak poly(A) signals in vivo leads to accumulation of unprocessed RNA that would otherwise be degraded (see Introduction).

PDD is distinct from the recently described pre-mRNA surveillance pathway in yeast, where inefficient polyadenylation leads to degradation of RNA that has been cleaved at the poly(A) site (Saguez et al. 2008). The pathway described by Saguez et al. is triggered by defects in proteins responsible for maturation and transport of the mRNA, whereas PDD appears to be a normal activity, not triggered by defects, that is directed by the poly(A) signal itself. Moreover, the substrate for PDD appears to be the nascent uncleaved RNA (Figs. 5 and 6), not post-transcriptional RNA that has already been cleaved at the poly(A) site. This is consistent with the properties of PDD in vivo, which indicate that it is slow cleavage, not slow polyadenylation, that leads to degradation. In vivo, most RNA-bearing weak poly(A) signals gets degraded; yet, much of this RNA can be rescued by placing a strong poly(A) signal downstream from the weak one (e.g., Peterson and Perry 1989; EnriquezHarris et al. 1991; Andrews and DiMaio 1993; Yang and Melera 1994). Since strong poly(A) signals process faster than weak ones (Chao et al. 1999), this observation is readily explained on the basis of a kinetic competition between the cleavage reactions. The rescue cannot be explained by competition between polyadenylation reactions on RNA that has already been cleaved at the weak site. Apparently, the PDD pathway is faster than cleavage at these weak sites but slower than cleavage at the strong sites, consistent with the results of Figure 5. Moreover, like cleavage itself, the PDD pathway operates on RNA that still contains an intact poly(A) signal.

Recently, an interesting example of experimentally induced PDD in vivo was reported (Fong et al. 2009). When cleavage at a strong poly(A) signal was suppressed by decapping the nascent transcript, the uncleaved RNA did 
not accumulate, but instead was degraded. The degradation was blocked, and the uncleaved RNA was allowed to accumulate by a point mutation in the poly(A) signal hexamer, confirming that the degradation caused by decapping was due to PDD.

Three mechanisms potentially responsible for PDD can be imagined. The first invokes the newly discovered endonuclease activity of the exosome (Lebreton et al. 2008; Schaeffer et al. 2009). Since the exosome most likely engages the nascent RNA cotranscriptionally (Andrulis et al. 2002; Custódio et al. 2007; Schmid and Jensen 2008) and interacts directly or indirectly with poly(A) polymerase (Burkard and Butler 2000), it is possible that the exosome itself is positioned at or near the poly(A) site, so as to cleave (and then to degrade) when cleavage directed by the $3^{\prime}$-end processing apparatus of a poly $(\mathrm{A})$ signal is slow.

A second mechanism that could conceivably account for PDD invokes the known multifunctionality of CPSF. For example, in mammalian histone RNA processing, cleavage by CPSF73 is not coupled to polyadenylation, and it is CPSF73 itself, not Xrn2, that exonucleolytically degrades the downstream RNA after cleavage (Yang et al. 2009). Perhaps, for poly(A) signals, one mode of CPSF73 cleavage is coupled to polyadenylation, whereas the other is coupled to degradation of the upstream RNA by the exosome. A kinetic competition between these two functionalities would predict high levels of degradation when authentic processing is slow.

A third mechanism that might account for PDD, and the one that we prefer, is suggested by the fact that some poly (A) signal-dependent (but cleavage-independent) release of downstream cassettes into the free RNA column fractions always occurs in our size exclusion chromatography experiments (data not shown). This observation is consistent with a discard mechanism in which transcripts that remain unprocessed are at risk of poly $(\mathrm{A})$ signaldependent release from the template, followed by $3^{\prime} \rightarrow 5^{\prime}$ exosomal degradation. Because there is no cleavage at the poly(A) site, this release could not be an Xrn2 exonucleasedependent event. However, the release could be mediated by the poly $(\mathrm{A})$ signal-dependent action of Pcf11 in the absence of cleavage (Sadowski et al. 2003; Qu et al. 2006). According to this mechanism, Xrn2-mediated termination would be coupled to processing, whereas Pcf11-mediated termination would be coupled to degradation. This mechanism would also explain how transcription could be subject to efficient $\operatorname{poly}(\mathrm{A})$ signal-dependent termination, even for $\operatorname{poly}(\mathrm{A})$ signals that cleave their transcripts very slowly and which also lack any special terminator elements (e.g., SV40 early in Orozco et al. 2002). This model is also consistent with the observation that knockdown of the nuclear exosome subunit PMScl100 in vivo increases the recovery of 3 '-end processed RNA for weak poly(A) signals (West and Proudfoot 2009).

\section{MATERIALS AND METHODS}

\section{Plasmid templates}

Reporter 1 (drawn to scale in Figs. 2A, 3A) is closely related (and identical in the region shown in Fig. $2 \mathrm{~A}$ ) to $\mathrm{pAP}\left\langle\mathrm{C}_{9}\right\rangle$ of Tran et al. (2001). The sequence between the cassettes, called $\mathrm{C}_{9}$, is a ninefold repeated snippet of eukaryotic DNA. The poly(A) signal mutant was derived from the wild type by site-directed mutagenesis (AATAAA $\rightarrow$ AgTAct). The elongation reference plasmid was derived from this mutant by removing Gs to lengthen the precassette (GGGCAAGCTG $\rightarrow$ aaaCAAtCTt) and by adding Gs to shorten the post-cassette (TCATTAT $\rightarrow$ TCgagAT). Reporter 2a (drawn to scale in Fig. 3A) is closely related (and identical from the post-cassette leftward) to the $\mathrm{pAP}\langle 117 \mathrm{cat}\rangle$ construct in Tran et al. (2001). The sequence between the cassettes is a piece of the prokaryotic chloramphenicol acetyl transferase gene. Reporter $2 b$ is presumed to be identical to 2a, having been derived, as a control, from the poly(A) signal mutant of $2 \mathrm{a}$ by back-mutagenesis. Reporter 3a (drawn to scale in Fig. 4A) was generated from Reporter 1 by cutting with StuI next to the promoter and inserting the following G-less cassette sequence $\left(5^{\prime} \rightarrow 3^{\prime}\right)$ : TGCCATACCCT TCCTCСATCTATACCACCCACTCTCСТTTCCTCATTATTCCT CCTATTATCTTCTCСТCTTCTCTCAGG. Reporter 3b, presumably identical to Reporter $3 \mathrm{a}$, is a control derived from the mutant of $3 \mathrm{a}$ by back-mutagenesis. Reporter 4 is identical to Reporter 3 except that the poly(A) signal was replaced by an identical length of DNA containing two AATAAA hexamers instead of a hexamer and a G/T-rich region (insert iii of Nag et al. 2006). The mutant version of this reporter, in which both hexamers are mutated, contains insert $\mathrm{v}$ from Nag et al. Reporter 5 is identical to Reporter 3 except that a SmaI-BamHI fragment containing the SV40 late poly(A) signal (Tran et al. 2001) replaces the HpaI-BamHI fragment of Reporter 3 that contains the SV40 early poly(A) signal. The mutant form of Reporter 5 contained AAgtAc in place of the wild-type poly(A) signal hexamer, AATAAA.

\section{In vitro transcription}

HeLa nuclear extract preparation and in vitro transcription were based on the procedures described by Tran et al. (2001). The concentrations of magnesium and citrate used in the assays were individually optimized for each extract preparation. Typically, 5-7 $\mathrm{mM}$ magnesium and 6-8 $\mathrm{mM}$ citrate were used, except for Figure $1 \mathrm{D}$, right (see legend). Where applicable, $\alpha$-amanitin and $3^{\prime}$-dATP were added as described in Rigo et al. (2005).

\section{Size exclusion chromatography}

Size exclusion chromatography of transcription reactions was done using a $3.1 \mathrm{~mL}(0.6 \mathrm{~cm} \times 11 \mathrm{~cm})$ column as described by Nag et al. (2006), except that $1 \%$ rather than $0.5 \%$ Sarkosyl was used for the experiments of Figure 1, B and C. Wild-type and mutant samples were transcribed in parallel, stopped as described by Nag et al. (2006), and then fractionated in succession on a size exclusion column. Since fractionating the wild-type and mutant samples in succession required that one of the samples be stored on ice for about 25 min before the fractionation, we alternated the column load order whenever experiments were repeated. We 
found that the initial extraction of column fractions should be with phenol rather than with TRIzol to avoid size bias in the recovery of the RNA. For Figure 2 the recovered RNA was then digested with RNase T1 to release the G-less cassettes, TRIzol extracted, and then loaded on the gel. For Figure 1 the column fractions were treated briefly with proteinase $\mathrm{K}$ before, and then with DNase I (rather than RNase T1) after the phenol extraction. Figure 6 was as for Figure 2 except that brief, successive RNase T1 and proteinase $\mathrm{K}$ treatments of the column fractions preceded the phenol extraction.

\section{Immobilized templates}

End-filling was used to generate biotinylated DNA for attaching to streptavidin-coated magnetic beads. Wild-type:mutant comparisons were made only between preparations made in parallel. All plasmid DNA was cut with EagI, and the end was filled using Klenow polymerase, dGTP, and Biotin-14-dCTP (Invitrogen). All DNAs were then cut a second time (Reporter 1 and the reference plasmid with AseI, and Reporter 2 with BsaI), and the resulting large biotinylated fragment from each plasmid was resolved using agarose gel electrophoresis and isolated using a Qiagen Gel Extraction kit. The Qiagen eluate for each reporter DNA was then mixed with eluate for reference DNA and added to an equal volume of magnetic beads suspended in kilobaseBINDER solution (1 $\mathrm{mg}$ beads per $10 \mu \mathrm{g}$ DNA). After rotation at room temperature for $\geq 10 \mathrm{~h}$, the beads were washed twice with Dynal B\&W Buffer, four times with $10 \mathrm{mM}$ Tris, $0.1 \mathrm{mM}$ EDTA ( $\mathrm{pH} 8$ ), and resuspended in $10 \mathrm{mM}$ Tris, $0.1 \mathrm{mM}$ EDTA (pH 8). About $30 \%$ of the input DNA became attached to the beads as estimated by restriction digestion and agarose gel analysis of a small aliquot of the preparation.

Following transcription, beads were removed by a 2-min magnetic selection, washed twice with nuclear extract buffer $\mathrm{D}$ (Tran et al. 2001) containing $0.5 \mathrm{mg} / \mathrm{mL}$ heparin and $0.5 \%$ Sarkosyl, and then the beads were treated with RNase T1 followed by TRIzol to recover the cassettes (Nag et al. 2006). RNA was precipitated with $20 \mu \mathrm{g}$ of Glycogen (Ambion) and $350 \mu \mathrm{L}$ of isopropanol, and the cassettes were separated on an $8 \%$ polyacrylamide gel.

Since the comparison of wild-type to mutant transcription for immobilized templates necessarily required comparing different immobilized template preparations to each other, we used transcription of the reference DNA (included on all the beads) to screen for equivalent performance of the immobilized templates as a condition for using any of the data from those preparations. Ideally, the elongation efficiencies, as indicated by the post/pre cassette ratios (see Fig. 2B; see the text), should be identical for the reference DNAs of all of the immobilized template preparations transcribed in parallel. But, in actuality, there was considerable variation among the preparations. Therefore, we included in our wild-type/mutant comparisons only immobilized template preparations that differed from each other by no more than $10 \%$ in their reference post/precassette ratios (for both pellet and supernatant). For the template preparations that survived this screen, the wildtype/mutant cassette ratio obtained for the reporter plasmids was then normalized to the wild-type/mutant ratio for their accompanying reference, as illustrated by the example in Figure 2B. Thus, the normalized $\mathrm{wt} / \mathrm{mt}$ ratio is given by the following expression: $\left[(\text { post } / \text { pre })_{\mathrm{wt}} /(\text { post } / \text { pre })_{\mathrm{mt}}\right]_{\text {reporter }} /\left[(\text { post } / \text { pre })_{\mathrm{wt}} /(\text { post } / \text { pre })_{\mathrm{mt}}\right]_{\text {reference }}$.

\section{ACKNOWLEDGMENTS}

We thank Frank Rigo and Anita Nag for important discussions during the course of this work, and the NIH and the Jonsson Cancer Center Foundation for support.

Received March 1, 2009; accepted September 22, 2009.

\section{REFERENCES}

Andrews EM, DiMaio D. 1993. Hierarchy of polyadenylation site usage by bovine papillomavirus in transformed mouse cells. J Virol 67: 7705-7710.

Andrulis ED, Werner J, Nazarian A, Erdjument-Bromage H, Tempst P, Lis JT. 2002. The RNA processing exosome is linked to elongating RNA polymerase II in Drosophila. Nature 420: 837-841.

Boireau S, Maiuri P, Basyuk E, de la Mata M, Knezevich A, PradetBalade B, Backer V, Kornblihtt A, Marcello A, Bertrand E. 2007. The transcriptional cycle of HIV-1 in real-time and live cells. J Cell Biol 179: 291-304.

Burkard KT, Butler JS. 2000. A nuclear $3^{\prime}-5^{\prime}$ exonuclease involved in mRNA degradation interacts with $\operatorname{Poly}(\mathrm{A})$ polymerase and the hnRNA protein npl3p. Mol Cell Biol 20: 604-616.

Calvo O, Manley JL. 2003. Strange bedfellows: Polyadenylation factors at the promoter. Genes \& Dev 17: 1321-1327.

Carswell S, Alwine JC. 1989. Efficiency of utilization of the simian virus 40 late polyadenylation site: Effects of upstream sequences. Mol Cell Biol 9: 4248-4258.

Chao LC, Jamil A, Kim SJ, Huang L, Martinson HG. 1999. Assembly of the cleavage and polyadenylation apparatus requires about 10 seconds in vivo and is faster for strong than for weak poly(A) sites. Mol Cell Biol 19: 5588-5600.

Connelly S, Manley JL. 1988. A functional mRNA polyadenylation signal is required for transcription termination by RNA polymerase II. Genes \& Dev 2: 440-452.

Custódio N, Vivo M, Antoniou M, Carmo-Fonseca M. 2007. Splicingand cleavage-independent requirement of RNA polymerase II CTD for mRNA release from the transcription site. J Cell Biol 179: 199-207.

Dantonel JC, Murthy KG, Manley JL, Tora L. 1997. Transcription factor TFIID recruits factor CPSF for formation of $3^{\prime}$ end of mRNA. Nature 389: 399-402.

Edwalds-Gilbert G, Prescott J, Falck-Pedersen E. 1993. 3' RNA processing efficiency plays a primary role in generating terminationcompetent RNA polymerase II elongation complexes. Mol Cell Biol 13: 3472-3480.

Enriquez-Harris P, Levitt N, Briggs D, Proudfoot NJ. 1991. A pause site for RNA polymerase II is associated with termination of transcription. EMBO J 10: 1833-1842.

Fong N, Ohman M, Bentley DL. 2009. Fast ribozyme cleavage releases transcripts from RNA polymerase II and aborts co-transcriptional pre-mRNA processing. Nat Struct Mol Biol 16: 916-922.

Glover-Cutter K, Kim S, Espinosa J, Bentley DL. 2008. RNA polymerase II pauses and associates with pre-mRNA processing factors at both ends of genes. Nat Struct Mol Biol 15: 71-78.

Gromak N, West S, Proudfoot NJ. 2006. Pause sites promote transcriptional termination of mammalian RNA polymerase II. Mol Cell Biol 26: 3986-3996.

Hara R, Selby CP, Liu M, Price DH, Sancar A. 1999. Human transcription release factor 2 dissociates RNA polymerases I and II stalled at a cyclobutane thymine dimer. J Biol Chem 274: 2477924786.

Hieronymus H, Yu MC, Silver PA. 2004. Genome-wide mRNA surveillance is coupled to mRNA export. Genes \& Dev 18: 2652 2662.

Kaneko S, Rozenblatt-Rosen O, Meyerson M, Manley JL. 2007. The multifunctional protein $\mathrm{p} 54 \mathrm{nrb} / \mathrm{PSF}$ recruits the exonuclease 
XRN2 to facilitate pre-mRNA $3^{\prime}$ processing and transcription termination. Genes \& Dev 21: 1779-1789.

Kim SJ, Martinson HG. 2003. Poly(A)-dependent transcription termination: Continued communication of the poly(A) signal with the polymerase is required long after extrusion in vivo. $J$ Biol Chem 278: 41691-41701.

Lebreton A, Tomecki R, Dziembowski A, Seraphin B. 2008. Endonucleolytic RNA cleavage by a eukaryotic exosome. Nature 456: 993 996.

Lian Z, Karpikov A, Lian J, Mahajan MC, Hartman S, Gerstein M, Snyder M, Weissman SM. 2008. A genomic analysis of RNA polymerase II modification and chromatin architecture related to $3^{\prime}$ end RNA polyadenylation. Genome Res 18: 1224-1237.

Liu M, Xie Z, Price DH. 1998. A human RNA polymerase II transcription termination factor is a SWI2/SNF2 family member. J Biol Chem 273: 25541-25544.

Marshall NF, Price DH. 1992. Control of formation of two distinct classes of RNA polymerase II elongation complexes. Mol Cell Biol 12: $2078-2090$.

Nag A, Narsinh K, Kazerouninia A, Martinson HG. 2006. The conserved AAUAAA hexamer of the poly(A) signal can act alone to trigger a stable decrease in RNA polymerase II transcription velocity. RNA 12: 1534-1544.

Nag A, Narsinh K, Martinson HG. 2007. The poly(A)-dependent transcriptional pause is mediated by CPSF acting on the body of the polymerase. Nat Struct Mol Biol 14: 662-669.

Orozco IJ, Kim SJ, Martinson HG. 2002. The poly(A) signal, without the assistance of any downstream element, directs RNA polymerase II to pause in vivo and then to release stochastically from the template. J Biol Chem 277: 42899-42911.

Park NJ, Tsao DC, Martinson HG. 2004. The two steps of poly(A)dependent termination, pausing and release, can be uncoupled by truncation of the RNA polymerase II CTD. Mol Cell Biol 24: 40924103.

Perkins KJ, Lusic M, Mitar I, Giacca M, Proudfoot NJ. 2008. Transcription-dependent gene looping of the HIV-1 provirus is dictated by recognition of pre-mRNA processing signals. Mol Cell 29: $56-68$.

Peterson ML, Perry RP. 1989. The regulated production of $\mu_{\mathrm{m}}$ and $\mu_{\mathrm{s}}$ mRNA is dependent on the relative efficiencies of $\mu_{s} \operatorname{poly}(\mathrm{A})$ site usage and the C $\mu 4$-to-M1 splice. Mol Cell Biol 9: 726-738.

Pillutla RC, Yue Z, Maldonado E, Shatkin AJ. 1998. Recombinant human mRNA cap methyltransferase binds capping enzyme/RNA polymerase IIo complexes. J Biol Chem 273: 21443-21446.

Prescott JC, Falck-Pedersen E. 1992. Varied poly(A) site efficiency in the adenovirus major late transcription unit. J Biol Chem 267: $8175-8181$.

Proudfoot NJ, Furger A, Dye MJ. 2002. Integrating mRNA processing with transcription. Cell 108: 501-512.

Qu X, Perez-Canadillas JM, Agrawal S, De Baecke J, Cheng H, Varani G, Moore C. 2006. The C-terminal domains of vertebrate CstF-64 and its yeast orthologue Rna15 form a new structure critical for mRNA $3^{\prime}$-end processing. J Biol Chem 282: 2101-2115.

Rigo F, Martinson HG. 2008. Functional coupling of last intron splicing and 3 '-end processing to transcription in vitro: The poly(A) signal couples to splicing before committing to cleavage. Mol Cell Biol 28: 849-862.

Rigo F, Martinson HG. 2009. Polyadenylation releases mRNA from RNA polymerase II in a process that is licensed by splicing. RNA 15: $823-836$.

Rigo F, Kazerouninia A, Nag A, Martinson HG. 2005. The RNA tether from the Poly(A) signal to the polymerase mediates coupling of transcription to cleavage and polyadenylation. Mol Cell 20: 733745.

Roth KM, Byam J, Fang F, Butler JS. 2009. Regulation of NAB2 mRNA 3 '-end formation requires the core exosome and the Trf4p component of the TRAMP complex. RNA 15: 1045-1058.

Rougemaille M, Dieppois G, Kisseleva-Romanova E, Gudipati RK, Lemoine S, Blugeon C, Boulay J, Jensen TH, Stutz F, Devaux F, et al. 2008. THO/Sub2p functions to coordinate $3^{\prime}$-end processing with gene-nuclear pore association. Cell 135: 308-321.

Ryner LC, Takagaki Y, Manley JL. 1989. Sequences downstream of AAUAAA signals affect pre-mRNA cleavage and polyadenylation in vitro both directly and indirectly. Mol Cell Biol 9: 17591771.

Sadowski M, Dichtl B, Hubner W, Keller W. 2003. Independent functions of yeast Pcfl1p in pre-mRNA $3^{\prime}$ end processing and in transcription termination. EMBO J 22: 2167-2177.

Saguez C, Schmid M, Olesen JR, Ghazy MA, Qu X, Poulsen MB, Nasser T, Moore C, Jensen TH. 2008. Nuclear mRNA surveillance in $\mathrm{THO} /$ sub2 mutants is triggered by inefficient polyadenylation. Mol Cell 31: 91-103.

Schaeffer D, Tsanova B, Barbas A, Reis FP, Dastidar EG, SanchezRotunno M, Arraiano CM, van Hoof A. 2009. The exosome contains domains with specific endoribonuclease, exoribonuclease and cytoplasmic mRNA decay activities. Nat Struct Mol Biol 16: $56-62$.

Schmid M, Jensen TH. 2008. Quality control of mRNP in the nucleus. Chromosoma 117: 419-429.

Shi Y, Di Giammartino DC, Taylor D, Sarkeshik A, Rice WJ, Yates JR 3rd, Frank J, Manley JL. 2009. Molecular architecture of the human pre-mRNA 3' processing complex. Mol Cell 33: 365376.

Tran DP, Kim SJ, Park NJ, Jew TM, Martinson HG. 2001. Mechanism of poly(A) signal transduction to RNA polymerase II in vitro. $\mathrm{Mol}$ Cell Biol 21: 7495-7508.

Uhlmann T, Boeing S, Lehmbacher M, Meisterernst M. 2007. The VP16 activation domain establishes an active mediator lacking CDK8 in vivo. J Biol Chem 282: 2163-2173.

Wahle E, Kühn U. 1997. The mechanism of $3^{\prime}$ cleavage and polyadenylation of eukaryotic pre-mRNA. Prog Nucleic Acid Res Mol Biol 57: 41-71.

West S, Proudfoot NJ. 2009. Transcriptional termination enhances protein expression in human cells. Mol Cell 33: 354-364.

$\mathrm{Wu}$ C, Alwine JC. 2004. Secondary structure as a functional feature in the downstream region of Mammalian polyadenylation signals. Mol Cell Biol 24: 2789-2796.

Yang H, Melera PW. 1994. A genetic polymorphism within the third poly(A) signal of the DHFR gene alters the polyadenylation pattern of DHFR transcripts in CHL cells. Nucleic Acids Res 22: $2694-2702$.

Yang XC, Sullivan KD, Marzluff WF, Dominski Z. 2009. Studies on the $5^{\prime}$ exonuclease and endonuclease activity of CPSF-73 in histone pre-mRNA processing. Mol Cell Biol 29: 31-42.

Yeung G, Choi LM, Chao LC, Park NJ, Liu D, Jamil A, Martinson HG. 1998. Poly(A)-driven and poly(A)-assisted termination: Two different modes of poly(A)-dependent transcription termination. Mol Cell Biol 18: 276-289.

Yonaha M, Proudfoot NJ. 2000. Transcriptional termination and coupled polyadenylation in vitro. EMBO J 19: 3770-3777.

Zhao J, Hyman L, Moore C. 1999. Formation of mRNA 3'ends in eukaryotes: Mechanism, regulation, and interrelationships with other steps in mRNA synthesis. Microbiol Mol Biol Rev 63: 405445 . 

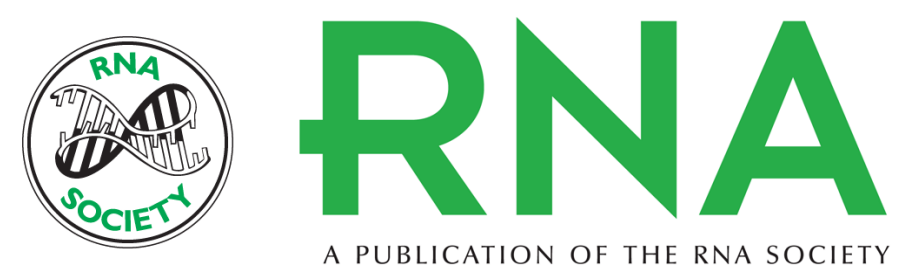

\section{$\operatorname{Poly}(A)$ signal-dependent degradation of unprocessed nascent transcripts accompanies poly $(A)$ signal-dependent transcriptional pausing in vitro}

Amir Kazerouninia, Benson Ngo and Harold G. Martinson

RNA 2010 16: 197-210 originally published online November 19, 2009

Access the most recent version at doi:10.1261/rna.1622010

References This article cites 54 articles, 34 of which can be accessed free at:

http://rnajournal.cshlp.org/content/16/1/197.full.html\#ref-list-1

\section{License}

Email Alerting

Receive free email alerts when new articles cite this article - sign up in the box at the Service top right corner of the article or click here.

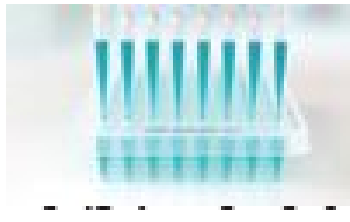

Providing Precise Solutions for your research.

To subscribe to $R N A$ go to:

http://rnajournal.cshlp.org/subscriptions 